\title{
Constitutive equation for polymer networks with phonon fluctuations
}

\author{
Hansen, Rasmus; Skov, Anne Ladegaard; Hassager, Ole
}

Published in:

Physical Review E

Link to article, DOI:

10.1103/PhysRevE.77.011802

Publication date:

2008

Document Version

Publisher's PDF, also known as Version of record

Link back to DTU Orbit

Citation (APA):

Hansen, R., Skov, A. L., \& Hassager, O. (2008). Constitutive equation for polymer networks with phonon fluctuations. Physical Review E, 77(1), 011802. https://doi.org/10.1103/PhysRevE.77.011802

\section{General rights}

Copyright and moral rights for the publications made accessible in the public portal are retained by the authors and/or other copyright owners and it is a condition of accessing publications that users recognise and abide by the legal requirements associated with these rights.

- Users may download and print one copy of any publication from the public portal for the purpose of private study or research.

- You may not further distribute the material or use it for any profit-making activity or commercial gain

- You may freely distribute the URL identifying the publication in the public portal

If you believe that this document breaches copyright please contact us providing details, and we will remove access to the work immediately and investigate your claim 


\title{
Constitutive equation for polymer networks with phonon fluctuations
}

\author{
Rasmus Hansen, ${ }^{1}$ Anne Ladegaard Skov, ${ }^{2}$ and Ole Hassager ${ }^{3}$ \\ ${ }^{1}$ Center for Fluid Dynamics at DTU, Technical University of Denmark, DK-2800 Kgs. Lyngby, Denmark \\ ${ }^{2}$ The Danish Polymer Center, Department of Chemical Engineering, Technical University of Denmark, \\ DK-2800 Kgs. Lyngby, Denmark \\ ${ }^{3}$ Center for Fluid Dynamics at DTU \& The Danish Polymer Center, Department of Chemical Engineering, \\ Technical University of Denmark, DK-2800 Kgs. Lyngby, Denmark
}

(Received 13 September 2007; published 14 January 2008)

Recent research by Xing et al. [Phys. Rev. Lett. 98, 075502 (2007)] has provided an expression for the Helmholtz free energy related to phonon fluctuations in polymer networks. We extend this result by constructing the corresponding nonlinear constitutive equation, usable for entirely general, volume conserving deformation fields. Constitutive equations for the sliplink model and the tube model are derived and the three models are examined by comparison with each other and with data from Xu and Mark [Rubber Chem. Technol. 63, 276 (1990)] and Wang and Mark [J. Polym. Sci., Part B: Polym. Phys. 30, 801 (1992)]. Elastic moduli are derived for the three models and compared with the moduli determined from the chemical stoichiometry. We conclude that the sliplink model and the phonon fluctuation model are relatively consistent with each other and with the data. The tube model seems consistent neither with the other models nor with the data.

DOI: 10.1103/PhysRevE.77.011802

PACS number(s): 61.41.+e

\section{INTRODUCTION}

A polymer gel is a three-dimensional network of polymer chains joined at a certain number of permanent connection sites, called crosslinks. Furthermore, a network usually contains entanglements, which can be regarded as temporary crosslinks. At the microscopic scale it resembles a liquid with a highly amorphous structure, however, it can sustain shear stresses without flowing, and hence behaves as an elastic solid. Since the 1940s, due to work done by Kuhn, Flory, and Treloar among others [4], it has been known that rubber elasticity is associated with a suppression of entropy by the imposed deformation. Kuhn derived the following expression for the elastic (Helmholtz) free energy, up to an additive function of volume and temperature, of a rubber in a homogeneous deformation [5]:

$$
A_{0}=\frac{3}{2} N_{c} k_{\mathrm{B}} T\left\langle|\mathbf{E} \cdot \boldsymbol{u}|^{2}\right\rangle_{\boldsymbol{u}}=\frac{1}{2} N_{c} k_{\mathrm{B}} T E_{i j}^{2},
$$

where $\langle\cdots\rangle_{u}$ denotes an average over the orientation of the three-dimensional unit vector $\boldsymbol{u}$, i.e., an isotropic distribution. $N_{c}$ is the number of chain segments between two neighboring connection sites, called partial chains, $k_{\mathrm{B}}$ the Boltzmann constant, $T$ the absolute temperature, and $E_{i j}$ $\equiv \partial x_{i} / \partial x_{j}^{\prime}$ the deformation gradient tensor, which is constant in a homogeneous deformation by definition. The 3 -vectors $\boldsymbol{x}^{\prime}$ and $\boldsymbol{x}$ denote the position of a material particle before and after, respectively, deformation. This result is based on two main assumptions: (1) The distribution function of the endto-end vector $\boldsymbol{r}$ of a partial chain is Gaussian. (2) The connection sites $r_{i}$ move affinely with the macroscopic deformation, i.e., $r_{i}=E_{i j} r_{j}^{\prime}$. We refer to Eq. (1) as the classical model, and to the corresponding network as an affine network. It has long been known that the classical model does not work well for large deformations.

As mentioned by Doi [5] the assumption of Gaussian statistics fails for large extensions, where the Langevin depen- dence of elongation on force becomes important. However, before this finite extensibility effect becomes important, two other neglected effects are dominant. These are the interactions between partial chains, and the fluctuations in the positions of the connection sites. To cope with entanglement interactions between polymer chains, Edwards and de Gennes introduced the tube model. This model implied another expression $[5,6]$ of the elastic free energy given by

$$
A_{t}=\frac{3}{2} N_{c} k_{\mathrm{B}} T \frac{Z^{2} a^{2}}{N b^{2}}\langle|\mathbf{E} \cdot \boldsymbol{u}|\rangle_{\boldsymbol{u}}^{2}=\frac{3}{2} N_{c} k_{\mathrm{B}} T Z\langle|\mathbf{E} \cdot \boldsymbol{u}|\rangle_{\boldsymbol{u}}^{2}
$$

Here $a$ is the tube radius, $N$ the number of Kuhn steps, $b$ the Kuhn step length, and $Z$ the number of elements of length $a$ spanning the central axis of the tube. Since $Z \propto N$, the prefactor increases linearly with $N$, which is known from experiments to be true. Note that the averaging in Eq. (2) occurs before the square, not after the square as in Eq. (1).

Ball et al. [7] introduced the concept of sliplinks, where entanglements are allowed to slide along the polymer strands, implying the elastic free energy

$$
\begin{aligned}
A_{s}= & A_{0}+\Delta A_{s}=\frac{1}{2} k_{\mathrm{B}} T N_{c} \sum_{i} \lambda_{i}^{2} \\
& +\frac{1}{2} k_{\mathrm{B}} T N_{s} \sum_{i}\left[\frac{(1+\eta) \lambda_{i}^{2}}{1+\eta \lambda_{i}^{2}}+\ln \left(1+\eta \lambda_{i}^{2}\right)\right] .
\end{aligned}
$$

Here $N_{c}$ and $N_{s}$ are the total number of crosslinks and sliplinks, respectively. $\lambda_{i}$ are Cartesian extension ratios (strains) and $\eta$ is a model specific parameter measuring the freedom of a link to slide compared with the freedom of movement of a chain. Ball et al. [7] recommended the value $\eta=0.2343$. Note that the second term, i.e., the contribution from the sliplinks, is added to the classical energy term, since the sliplink nature describes entanglements only.

Fluctuations in the positions of the connection sites have historically been a great barrier towards a precise and physi- 
cally reasonable model of rubber elasticity, also working for large deformations. Rubinstein and Panyukov [8] have combined the ideas of the confining tube and sliplink models to a slip-tube model. More recent research by Xing et al. [1] provides another modification term to the classical model, based on incompressible phonon fluctuations of the polymer network:

$$
\Delta A_{p}=\frac{4 \pi}{\xi^{3}} V k_{\mathrm{B}} T\left\langle\ln \left(C_{\alpha \beta} u_{\alpha} u_{\beta}\right)\right\rangle_{u} .
$$

In this expression $\xi$ is the typical mesh size of the polymer network, $V$ the volume of the material, and $C_{\alpha \beta}$ the Cauchy strain tensor defined below. In addition to deriving this general result, Xing et al. carry out the averaging in Eq. (4) for uniaxially and biaxially deformed systems. They also demonstrate a very encouraging comparison between their theory and data for uniaxial and biaxial stretching of crosslinked networks.

\section{CONSTITUTIVE EQUATIONS}

In this section we derive general expressions for the stress tensor, $\sigma_{i j}$, based on the models from Sec. I. We also introduce the elastic moduli as five independent fitting parameters, $G_{0}^{s}, G_{0}^{p}, G_{s}, G_{p}$, and $G_{t}$, for the three considered models. Following Bird et al. [9] we have the general expression for the stress in terms of the free energy density $f$,

$$
\sigma_{i j}=E_{i n} \frac{\partial f}{\partial E_{j n}} .
$$

The hydrostatic pressure $p$, specified by some imposed boundary condition, contributes with an isotropic tensor $p \boldsymbol{\delta}$, where $\boldsymbol{\delta}$ is the unit tensor.

When inserting Eq. (1) into Eq. (5) we get the constitutive equation for the classical model,

$$
\sigma_{i j}^{\text {classical }}=G_{0} E_{i n} E_{j n} .
$$

The elastic modulus is $G_{0}=n_{c} k_{\mathrm{B}} T$, where we have defined the density of partial chains $n_{c} \equiv N_{c} / V$. Since stresses obtained by the sliplink model (Sec. II B) and the phonon fluctuation model (Sec. II C) are added to the classical contribution, we define, respectively, $G_{0}^{s}$ and $G_{0}^{p}$.

\section{A. Tube model}

The constitutive equation for the tube model is obtained by inserting Eq. (2) into Eq. (5). Following Nielsen et al. [10] we get

$$
\begin{aligned}
\sigma_{i j}^{\text {tube }} & =3 n_{c} k_{\mathrm{B}} T Z\langle|\mathbf{E} \cdot \boldsymbol{u}|\rangle_{\boldsymbol{u}}\left\langle\frac{E_{i m} u_{m} E_{j n} u_{n}}{|\mathbf{E} \cdot \boldsymbol{u}|}\right\rangle_{\boldsymbol{u}} \\
& =\frac{15}{4} G_{t}\langle\mathbf{E} \cdot \boldsymbol{u} \mid\rangle_{\boldsymbol{u}}\left\langle\frac{E_{i m} u_{m} E_{j n} u_{n}}{|\mathbf{E} \cdot \boldsymbol{u}|}\right\rangle_{\boldsymbol{u}},
\end{aligned}
$$

where the elastic modulus $G_{t}=(4 / 5) n_{c} k_{\mathrm{B}} T Z$.

\section{B. Sliplink model}

The constitutive equation corresponding to the sliplink energy term from Eq. (3) is derived next. First, we remember that the nonlinear Finger strain tensor $B_{i j}$ and the Cauchy strain tensor $C_{i j}=B_{i j}^{-1}$ are defined as follows:

$$
\begin{aligned}
& B_{i j}=E_{i n} E_{j n}, \\
& C_{i n} B_{n j}=\delta_{i j} .
\end{aligned}
$$

The Finger strain tensor $B_{i j}$ is in some literature called the left Cauchy-Green tensor, but the Cauchy strain tensor $C_{i j}$ is not the same as the right Cauchy-Green tensor.

We also introduce the two strain invariants

$$
\begin{gathered}
I_{1}=B_{i i}=\lambda_{1}^{2}+\lambda_{2}^{2}+\lambda_{3}^{2}, \\
I_{2}=C_{i i}=\lambda_{1}^{-2}+\lambda_{2}^{-2}+\lambda_{3}^{-2},
\end{gathered}
$$

and the incompressibility condition

$$
\lambda_{1} \lambda_{2} \lambda_{3}=1 \text {. }
$$

Using the chain rule, we obtain

$$
\begin{gathered}
E_{i n} \frac{\partial}{\partial E_{j n}} I_{1}=2 B_{i j}, \\
E_{i n} \frac{\partial}{\partial E_{j n}} I_{2}=-2 C_{i j} .
\end{gathered}
$$

Hence in general for some function $F\left(I_{1}, I_{2}\right)$ we have

$$
E_{i n} \frac{\partial}{\partial E_{j n}} F\left(I_{1}, I_{2}\right)=2 \frac{\partial F}{\partial I_{1}} B_{i j}-2 \frac{\partial F}{\partial I_{2}} C_{i j} .
$$

Denoting the energy density $\Delta A_{s} / V$ by $f_{\text {slip }}$ and the number density $N_{s} / V$ by $n_{s}$, we have from Eq. (3)

$$
\begin{aligned}
\frac{f_{\text {slip }}}{k_{\mathrm{B}} T n_{s}}= & \frac{(1+\eta)}{2}\left[\frac{\lambda_{1}^{2}}{1+\eta \lambda_{1}^{2}}+\frac{\lambda_{2}^{2}}{1+\eta \lambda_{2}^{2}}+\frac{\lambda_{3}^{2}}{1+\eta \lambda_{3}^{2}}\right] \\
& +\frac{1}{2} \ln \left[\left(1+\eta \lambda_{1}^{2}\right)\left(1+\eta \lambda_{2}^{2}\right)\left(1+\eta \lambda_{3}^{2}\right)\right] .
\end{aligned}
$$

Rewriting in terms of strain invariants, we obtain

$$
\begin{aligned}
\frac{f_{\text {slip }}}{k_{\mathrm{B}} T n_{s}}= & \frac{(1+\eta)}{2} \frac{I_{1}+2 \eta I_{2}+3 \eta^{2}}{1+\eta I_{1}+\eta^{2} I_{2}+\eta^{3}} \\
& +\frac{1}{2} \ln \left(1+\eta I_{1}+\eta^{2} I_{2}+\eta^{3}\right) .
\end{aligned}
$$

From Eq. (15) we can write

$$
\sigma_{i j}^{s l i p}=2 \frac{\partial f_{s l i p}}{\partial I_{1}} B_{i j}-2 \frac{\partial f_{s l i p}}{\partial I_{2}} C_{i j} .
$$

By differentiation we obtain

$$
\begin{aligned}
& \frac{\partial}{\partial I_{1}} \frac{f_{\text {slip }}}{k_{\mathrm{B}} T n_{s}}=\frac{(1+\eta)}{2} \frac{1-\eta^{2} I_{2}-2 \eta^{3}}{X^{2}}+\frac{\eta}{2 X}, \\
& \frac{\partial}{\partial I_{2}} \frac{f_{\text {slip }}}{k_{\mathrm{B}} T n_{s}}=\frac{(1+\eta)}{2} \frac{2 \eta+\eta^{2} I_{1}-\eta^{4}}{X^{2}}+\frac{\eta^{2}}{2 X},
\end{aligned}
$$

where $X \equiv 1+\eta I_{1}+\eta^{2} I_{2}+\eta^{3}$. 
Specializing to shear deformation of shear magnitude $\gamma$, we find

$$
\begin{gathered}
\sigma_{x y}=n_{s} k_{\mathrm{B}} T \gamma \frac{(1+\eta)^{2}+\eta^{2} \gamma^{2}}{\left[(1+\eta)^{2}+\eta \gamma^{2}\right]^{2}}, \\
N_{1}=\sigma_{x x}-\sigma_{y y}=\gamma \sigma_{x y}, \\
N_{2}=\sigma_{y y}-\sigma_{z z}=-n_{s} k_{\mathrm{B}} T \gamma^{2} \eta \frac{2(1+\eta)+\eta \gamma^{2}}{\left[(1+\eta)^{2}+\eta \gamma^{2}\right]^{2}},
\end{gathered}
$$

as obtained by Oberdisse et al. [11]. From the zero strain limit of the shear stress expression, we obtain the elastic modulus

$$
G_{s}=\frac{n_{s} k_{\mathrm{B}} T}{(1+\eta)^{2}} .
$$

\section{Phonon fluctuation model}

To obtain the general constitutive equation for polymer networks with phonon fluctuations, one must insert the free energy from Eq. (4) into Eq. (5).

$$
\begin{aligned}
\sigma_{i j} & =2 \pi k_{\mathrm{B}} T \xi^{-3}\left\langle E_{i n} \frac{\partial}{\partial E_{j n}} \ln \left(C_{\alpha \beta} u_{\alpha} u_{\beta}\right)\right\rangle_{u} \\
& =2 \pi k_{\mathrm{B}} T \xi^{-3}\left\langle\begin{array}{c}
\frac{\partial}{\partial E_{j n}} C_{p q} u_{p} u_{q} \\
E_{\alpha \beta} u_{\alpha} u_{\beta}
\end{array}\right\rangle_{u} .
\end{aligned}
$$

The derivative in the numerator is carried out:

$$
E_{i n} \frac{\partial}{\partial E_{j n}} C_{p q}=E_{i n} \frac{\partial}{\partial E_{j n}} B_{p q}^{-1}=-C_{p j} \delta_{i q}-C_{j q} \delta_{p i} .
$$

Hence

$$
\begin{aligned}
E_{\text {in }} \frac{\partial}{\partial E_{j n}} C_{p q} u_{p} u_{q} & =-C_{p j} \delta_{i q} u_{p} u_{q}-C_{j q} \delta_{p i} u_{p} u_{q} \\
& =-C_{p j} u_{p} u_{i}-C_{j q} u_{q} u_{i} .
\end{aligned}
$$

The Cauchy strain tensor is symmetric by construction, which implies

$$
E_{i n} \frac{\partial}{\partial E_{j n}} C_{p q} u_{p} u_{q}=-2 C_{i p} u_{p} u_{j}=-2 \mathbf{C} \cdot \boldsymbol{u u} .
$$

Recognizing the denominator inside the brackets in Eq. (25) as the double dot product, we arrive at the central result of the paper; the stress-strain constitutive equation corresponding to incompressible phonon fluctuations.

$$
\sigma_{i j}=-4 \pi k_{\mathrm{B}} T \xi^{-3}\left\langle\frac{C_{i p} u_{p} u_{j}}{C_{\alpha \beta} u_{\alpha} u_{\beta}}\right\rangle_{u}=-4 \pi k_{\mathrm{B}} T \xi^{-3}\left\langle\frac{\mathbf{C} \cdot \boldsymbol{u u}}{\mathbf{C}: \boldsymbol{u u}}\right\rangle_{u} .
$$

This expression is evaluated numerically in simple shear for $N_{1}, N_{2}$, and $\sigma_{x y}$, see Fig. 1. To second order in the shear $\gamma$, the normal stress ratio $\Psi=N_{2} / N_{1}=-5 / 7$.
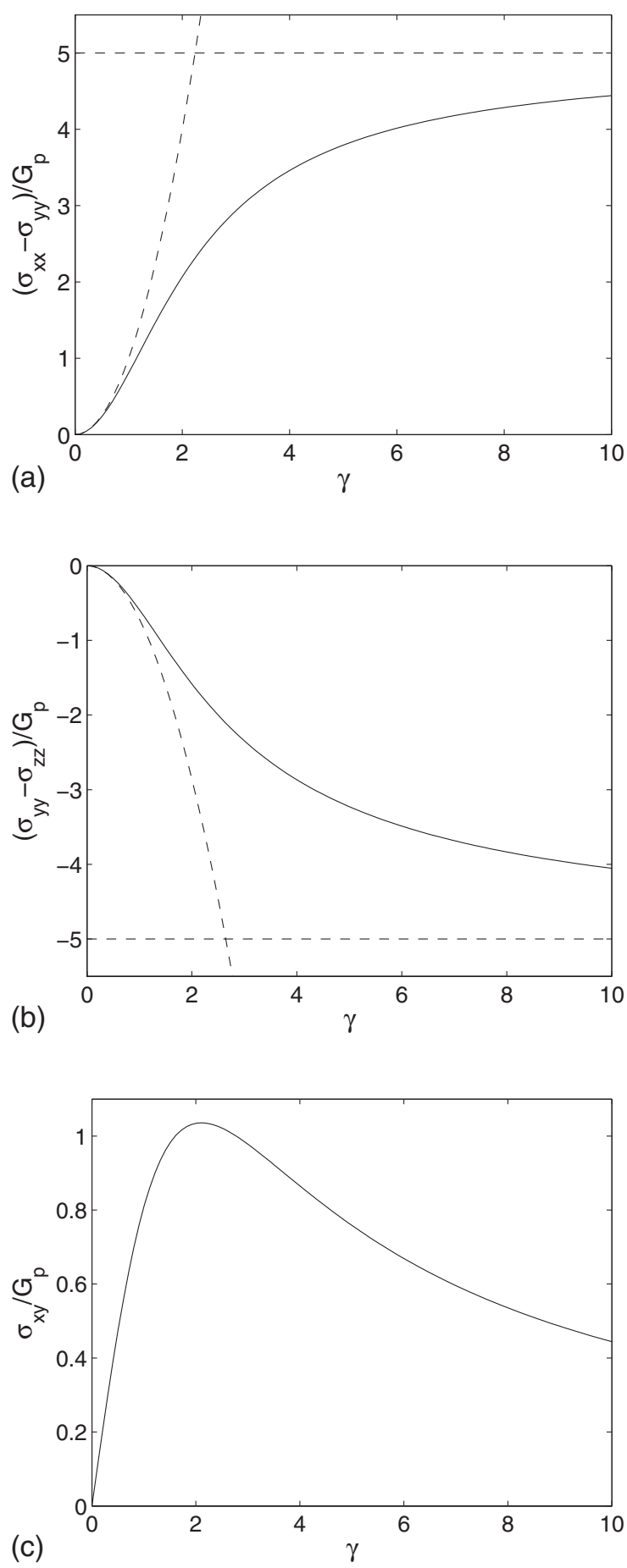

FIG. 1. Dimensionless normal stress differences $N_{1} / G_{p}$ and $N_{2} / G_{p}$ and shear stress $\sigma_{x y} / G_{p}$ from phonon fluctuations in simple shear deformation as a function of the shear magnitude $\gamma$. Numerical evaluations of Eq. (29) are plotted along with second order expansions, $N_{1} / G_{p} \approx \gamma^{2}$ and $N_{2} / G_{p} \approx-5 / 7 \gamma^{2}$, and asymptotics, $N_{1} / G_{p} 5$ and $N_{2} / G_{p}-5$ (dashed lines).

We now proceed to derive the elastic modulus $G_{p}$ for the phonon fluctuation model. For infinitesimal shear strain we set

$$
C_{m n}=\delta_{m n}-\epsilon_{m n},
$$


and expand to first order in $\epsilon_{m n}$ :

$$
\begin{aligned}
\left\langle\frac{C_{i p} u_{p} u_{j}}{C_{\alpha \beta} u_{\alpha} u_{\beta}}\right\rangle_{u} & =\left\langle\frac{u_{i} u_{j}-\epsilon_{i p} u_{p} u_{j}}{1-\epsilon_{\alpha \beta} u_{\alpha} u_{\beta}}\right\rangle_{u} \\
& =\left\langle u_{i} u_{j}+\epsilon_{\alpha \beta} u_{\alpha} u_{\beta} u_{i} u_{j}-\epsilon_{i p} u_{p} u_{j}\right\rangle_{u}+O\left(\epsilon_{i j}^{2}\right) .
\end{aligned}
$$

Using the identities

$$
\begin{gathered}
\left\langle u_{\alpha} u_{\beta}\right\rangle_{u}=\frac{1}{3} \delta_{\alpha \beta}, \\
\left\langle u_{\alpha} u_{\beta} u_{i} u_{j}\right\rangle_{u}=\frac{1}{15}\left(\delta_{\alpha \beta} \delta_{i j}+\delta_{\alpha i} \delta_{\beta j}+\delta_{\alpha j} \delta_{\beta j}\right)
\end{gathered}
$$

we get

$$
\left\langle\frac{C_{i p} u_{p} u_{j}}{C_{\alpha \beta} u_{\alpha} u_{\beta}}\right\rangle_{u}=\frac{1}{3} \delta_{i j}-\frac{1}{5} \epsilon_{i j}+O\left(\epsilon_{i j}^{2}\right) .
$$

Hence the elastic modulus is given by $G_{p}=4 \pi k_{\mathrm{B}} T \xi^{-3} / 5$. Note that $G_{p}$ and $G_{0}$ are not entirely independent, since the mesh size $\xi$ depends on the crosslink density $n_{c}$. However, following Xing et al. [1], we use both as free fitting parameters.

\section{STRESS-STRAIN CURVES AND DATA}

Uni- and bi-axial deformation is characterized by the strain $\lambda$, where $\lambda>1$ and $\lambda<1$ correspond to uni- and biaxial deformation, respectively. The deformation gradient tensor is given by $\mathbf{E}=\operatorname{diag}\left(\lambda^{-1 / 2}, \lambda^{-1 / 2}, \lambda\right)$. In uniaxial deformation $(\lambda>1)$ a sphere is deformed into a prolate ellipsoid, while in biaxial deformation $(\lambda<1)$ the sphere is deformed into an oblate ellipsoid.

In simple shear the characterization parameter is $\gamma$, and the deformation gradient tensor is given by

$$
\mathbf{E}=\left(\begin{array}{lll}
1 & \gamma & 0 \\
0 & 1 & 0 \\
0 & 0 & 1
\end{array}\right)
$$

Planar elongation is characterized by the parameter $\alpha$, and the deformation gradient tensor is given by $\mathbf{E}$ $=\operatorname{diag}\left(\alpha, 1, \alpha^{-1}\right)$. The considered material is assumed incompressible in all the considered models, i.e., det $\mathbf{E}=1$.

In order to obtain parameters, we make a fit of the three considered models in uni- and bi-axial deformation to data from $\mathrm{Xu}$ and Mark [2], in the Mooney-Rivlin plot in Fig. 2 (a). To test the theories, we compare the models with data from the same network [3] in planar elongation, using the same set of parameters, in Fig. 2 (b). It is important to notice that the data has a slight inconsistency in the modulus but the deviation is within experimental uncertainty (5\%). In both figures the classical contribution only translates the curves vertically. It is noteworthy how similar the phonon fluctuation model and the sliplink model are. They both contain the essence of the deviation from classical behavior, possibly within experimental error. The tube model is unable to fit the data.

For comparison, we have given also in Table I the value $G_{0}=0.114 \times 10^{6} \mathrm{~Pa}$ denoted "chemistry." This value is com-
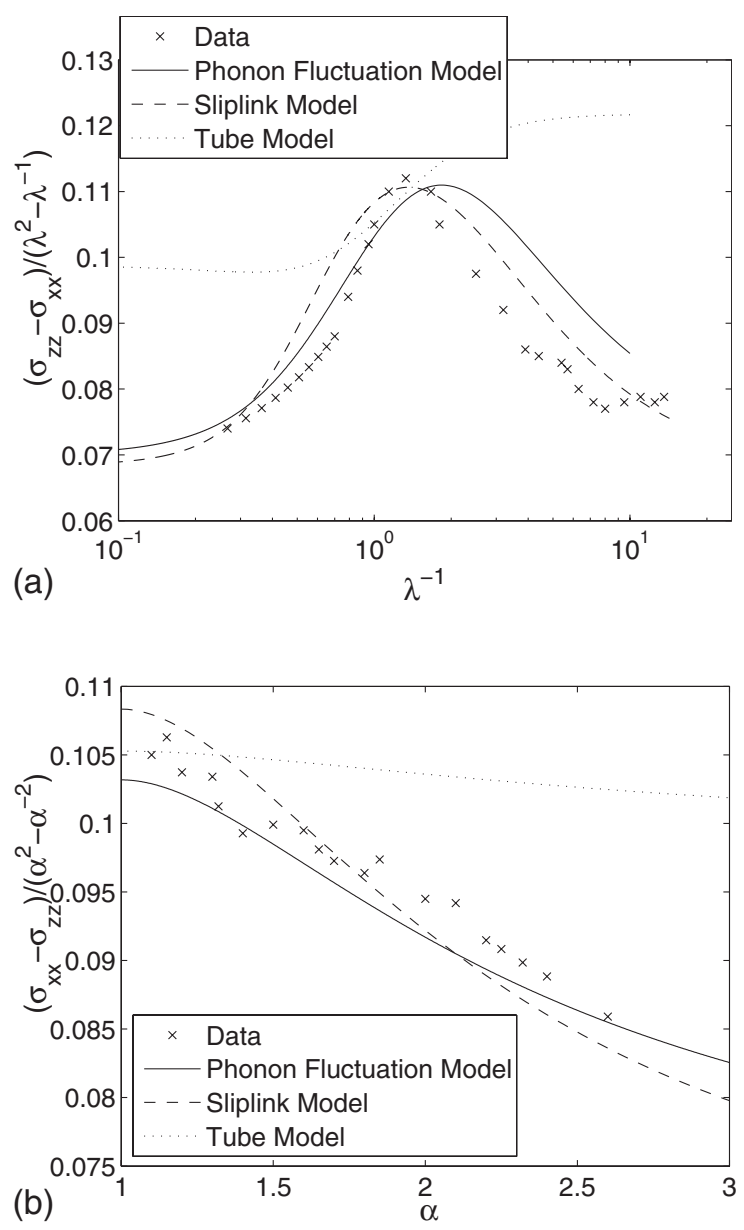

FIG. 2. (a) Fit of the phonon fluctuation model, the sliplink model, and the tube model to data for uni- and bi-axial deformation. The temperature is $T=298 \mathrm{~K}$. (b) Comparison of the three models with data from the same network in planar elongation, using the same set of parameters. Data from Xu and Mark [2] and Wang and Mark [3] (unit: $10^{6} \mathrm{~Pa}$ ). In order for the two data sets to predict the same modulus for $\alpha=\lambda=1$ we have scaled the data for planar elongation with a factor of 0.9545 . This corresponds to a temperature variation of approximately $14 \mathrm{~K}$. Parameters are presented in Table I.

puted from the expression $G_{0}=n_{c} k_{\mathrm{B}} T$ with $n_{c}$ obtained from the stoichiometry given by $\mathrm{Xu}$ and Mark [2]. The value closely predicts the total modulus of the network. However, the classical model alone clearly does not describe the nonlinear elasticity of the network. The additional contributions

TABLE I. Parameter values used to fit models to data in Fig. 2 (unit: $10^{6} \mathrm{~Pa}$ ).

\begin{tabular}{lccc}
\hline \hline Model & Elastic modulus & $\begin{array}{c}\text { Affine } \\
\text { (classical) }\end{array}$ & Additional \\
\hline Tube & $G_{t}=4 n_{c} k_{\mathrm{B}} T Z / 5$ & & 0.1053 \\
Sliplink & $G_{s}=n_{s} k_{\mathrm{B}} T /(1+\eta)^{2}$ & 0.0683 & 0.0400 \\
Phonon & $G_{p}=4 \pi k_{\mathrm{B}} T \xi^{-3} / 5$ & 0.0700 & 0.0322 \\
Chemistry & $G_{0}=0.114$ & & \\
\hline \hline
\end{tabular}



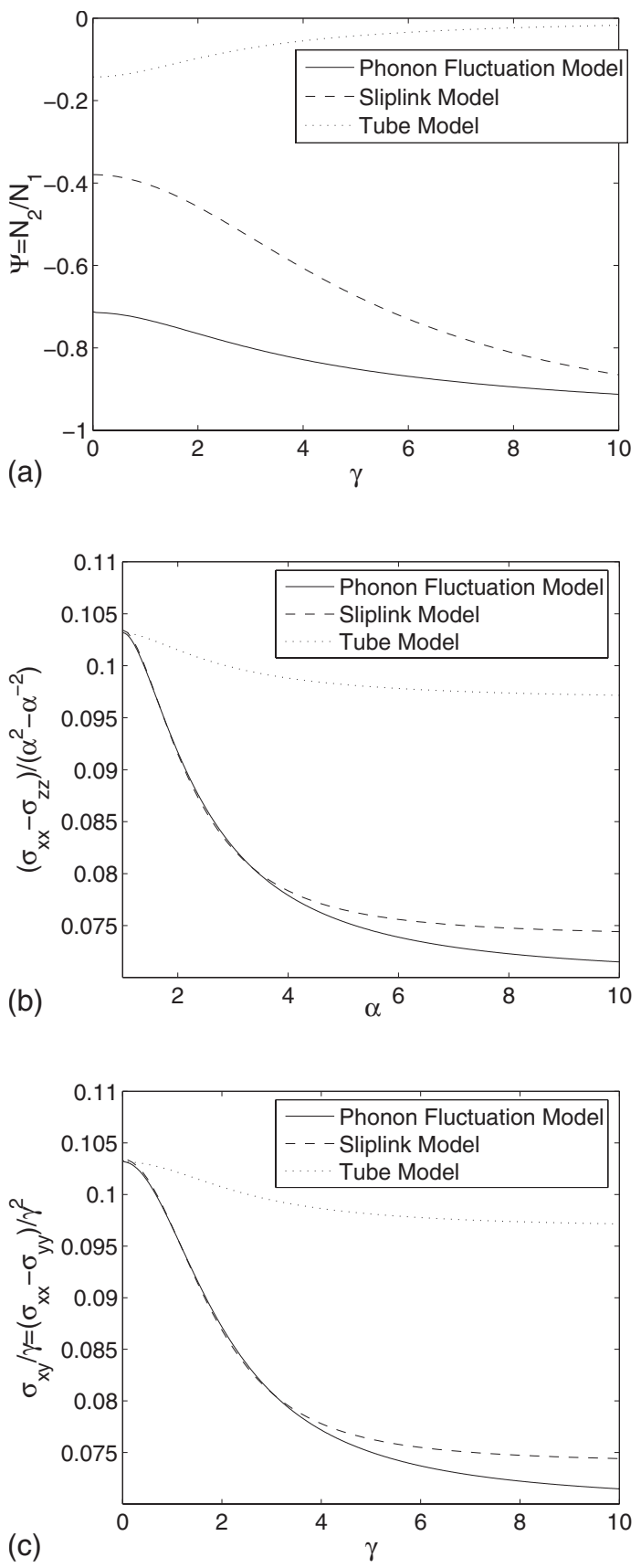

FIG. 3. (a) Normal stress ratio as a function of shear $\gamma$. For the classical model $\Psi=0$. (b-c) Comparison of the phonon fluctuation model, the sliplink model, and the tube model in (planar elongation/ simple shear). Parameters for the phonon fluctuation model are taken from the data fit, see Table I, and the two other models are fitted to the phonon fluctuation model.

from either the sliplink model or the phonon fluctuation model greatly improve the nonlinear behavior, whereby almost a perfect fit is obtained. When the additional terms are added it becomes necessary to reduce the classical contribution. The values for $G_{0}^{s}$ and $G_{0}^{p}$ in Table I are closer to the values predicted by the phantom network model [2], which for the tetrafunctional network is exactly one-half the affine network expression. It seems less obvious how the parameter $Z$ in the tube model can be predicted from the stoichiometry $[12,13]$. The molar mass between crosslinks is $21 \mathrm{~kg} / \mathrm{mol}$, while the entanglement molar mass is $13 \mathrm{~kg} / \mathrm{mol}$ [14]. This could suggest a number $Z$ in the range 1 to 2 , which is in reasonable agreement with Table I. Keep in mind, however, the nonlinear behavior of the tube model is inconsistent with the data.

In closing we compare the three models in large deformations outside the experimentally investigated range. Figure 3 (a) shows the normal stress ratio $\Psi=N_{2} / N_{1}$ in simple shear. In (b) we compare the models in planar elongation, fitting the tube model and the sliplink model to the phonon fluctuation model. In (c) we compare the models in simple shear. The sliplink model and the phonon fluctuation model produce very similar results. The tube model deviates markedly from the two other models. The existence of the second normal stress difference is well-documented for polymeric liquids, and the tube model has formed the basis for the pioneering Doi-Edwards model for entangled polymer melts $[5,6]$.

Planar elongation and simple shear differ only by a rotation of coordinates, i.e., the stress components for planar elongation $[\cdots]_{p}$ and simple shear $[\cdots]_{s}$ are related by the formula

$$
\frac{\left[\sigma_{x x}-\sigma_{z z}\right]_{p}}{\alpha^{2}-\alpha^{-2}}=\frac{\left[\sigma_{x y}\right]_{s}}{\gamma}=\frac{\left[\sigma_{x x}-\sigma_{y y}\right]_{s}}{\gamma^{2}},
$$

provided that the two deformation parameters are related by

$$
\alpha^{2}=1+1 / 2 \gamma^{2}+\gamma \sqrt{1+1 / 4 \gamma^{2}} .
$$

This result is obtained by diagonalizing the Finger strain tensor $B_{i j}$ for simple shear.

\section{CONCLUSIONS}

The phonon fluctuation model is capable of fitting simultaneously uniaxial, biaxial, and planar extension. At the same time, however, the data may be fitted equally well by the sliplink model, which is based on very different physical ideas. The phonon fluctuation model therefore offers an alternative physical explanation of the deviations from the classical behavior in rubber elasticity. Both models require that the modulus from the classical term, as well as the moduli from the additional terms, are retained as free fitting parameters.

The tube model does provide clear deviations from classical behavior in a Mooney plot. Uniaxial extension and planar elongation are qualitatively correct, while the model fails to give a maximum in biaxial compression.

\section{ACKNOWLEDGMENTS}

A. L. Skov is grateful for the financial support from the Danish Research Council for Technology and Production Sciences, the Danish Polymer Center is supported by a grant from The Danish Research Council for Technology and Production Sciences, and Fluid.DTU is supported from the Danish National Research Foundation. 
[1] X. Xing, P. M. Goldbart, and L. Radzihovsky, Phys. Rev. Lett. 98, 075502 (2007).

[2] P. Xu and J. E. Mark, Rubber Chem. Technol. 63, 276 (1990).

[3] S. Wang and J. E. Mark, J. Polym. Sci., Part B: Polym. Phys. 30, 801 (1992).

[4] L. R. G. Treloar, The Physics of Rubber Elasticity (Clarendon Press, Oxford, 1975).

[5] M. Doi, Introduction to Polymer Physics (Oxford University Press, New York, 1995).

[6] M. Doi and S. F. Edwards, The Theory of Polymer Dynamics (Oxford University Press, New York, 1986).

[7] R. C. Ball, M. Doi, S. F. Edwards, and M. Warner, Polymer 22, 1010 (1981).

[8] M. Rubinstein and S. Panyukov, Macromolecules 35, 6670 (2002).
[9] R. B. Bird, R. C. Armstrong, and O. Hassager, Dynamics of Polymeric Liquids, Vol. 1: Fluid Mechanics (Wiley, New York, 1987).

[10] J. K. Nielsen, H. K. Rasmussen, M. Denberg, K. Almdal, and O. Hassager, Macromolecules 39, 8844 (2006).

[11] J. Oberdisse, G. Ianniruberto, F. Greco, and G. Marrucci, Rheol. Acta 46, 95 (2006).

[12] A. L. Larsen, K. Hansen, P. Sommer-Larsen, O. Hassager, A. Bach, S. Ndoni, and M. Joergensen, Macromolecules 36, 10063 (2003).

[13] A. L. Larsen, P. Sommer-Larsen, and O. Hassager, e-Polymers 050 (2004).

[14] L. J. Fetters, D. J. Lohse, D. Richter, T. A. Witten, and A. Zirkel, Macromolecules 27, 4639 (1994). 